\title{
ORIGINAL RESEARCH \\ Cervical Ribs: A Common Variant Overlooked in CT Imaging
}

\author{
V.G. Viertel \\ J. Intrapiromkul \\ F. Maluf \\ N.V. Patel \\ W. Zheng \\ F. Alluwaimi \\ M.J. Walden \\ A. Belzberg \\ D.M. Yousem
}

BACKGROUND AND PURPOSE: Cervical ribs are congenital variants that are known to cause TOS or brachial plexopathy in up to $10 \%$ of the affected individuals. We investigated how often cervical ribs are present on cervical spine CT scans to determine the incidence in humans and the percentage of reported cervical ribs.

MATERIALS AND METHODS: Cervical spine CT scans and the reports of 3404 consecutive adult patients were retrospectively reviewed to determine the presence of cervical ribs and whether they had been reported.

RESULTS: Cervical ribs were found in $2.0 \%$ (67/3404) of the population. Of the 67 patients with cervical ribs, $27(40.3 \%)$ had bilateral ribs. The prevalence of cervical ribs in women was twice that in men, $2.8 \%$ (39/1414) versus $1.4 \%$ (28/1990). Although African Americans accounted for $50.1 \%$ (1706/3404) and whites, $41.2 \%$ (1402/3404) of the patient population, African Americans were $70.1 \%$ $(47 / 67)$ of patients with cervical ribs, whereas whites were $26.9 \%$ (18/67). Radiologists commented on $25.5 \%(24 / 94)$ of the cervical ribs in $25.4 \%(27 / 67)$ of patients.

CoNCLUSIONS: The prevalence of cervical ribs in the human population has been a source of uncertainty due to the degree of difficulty that comes in detecting this often subtle congenital variation. In our sample, the prevalence was $2.0 \%$ of patients. Our study determined that cervical ribs are underreported in patients undergoing cervical spine CT. Given the potential clinical implications of these anatomic variants, neuroradiologists must be more meticulous in identifying cervical ribs when reviewing cervical spine CT scans.

ABBREVIATION: TOS $=$ thoracic outlet syndrome
$\mathbf{T}$ he development of the spine in a fetus is based on the formation of groups of mesenchymal cells from the mesoderm on either side of the neural tube. These cells form somites, which later become vertebrae postossification. ${ }^{1}$ Typical development leads to 7 cervical vertebrae followed by 12 rib-bearing thoracic vertebrae. Mutations in Hox genes have been shown to cause the development of cervical ribs from the costal or ventral processes of the primitive vertebral arches. ${ }^{2,3}$ These can be associated with stillbirths and childhood malignancies. ${ }^{1,4}$

Cervical ribs are relatively common anomalies with a wide range of reported incidences $(0.05 \%-3.0 \%)$ based on series using radiographs. ${ }^{2,5,6}$ They are associated with TOS in approximately $10 \%$ of the affected population. ${ }^{2,7}$

This study was conceived for 2 main reasons: First, cervical ribs are abnormalities that may cause an uncomfortable and often painful syndrome in humans; surgery, in which the supernumerary rib is removed, is often the only relief for affected individuals. ${ }^{7,8}$ Second, there is an apparent discrepancy in the prevalence of the variant in the literature and its rate of detection in our routine radiology practice. Periodic investigations of the reporting success of various abnormalities and normal variants ensure that radiologists are meticulous and thorough

Received January 25, 2012; accepted after revision February 21.

From The Russell H. Morgan Department of Radiology and Radiological Sciences (V.G.V., J.I., F.M., N.V.P., W.Z., F.A., M.J.W., D.M.Y.) and Department of Neurosurgery (A.B.), The Johns Hopkins Medical Institutions, Baltimore, Maryland.

Please address correspondence to David M. Yousem, MD, The Russell H. Morgan Department of Radiology and Radiological Sciences, The Johns Hopkins Medical Institutions, 600 N Wolfe St, Phipps B-100F, Baltimore, MD 21287; e-mail: dyousem1@jhu.edu http://dx.doi.org/10.3174/ajnr.A3143 in their efforts in reading scans. Previous studies with similar objectives have found underreporting to be a significant problem in radiology. For example, Anwar et $\mathrm{al}^{9}$ found that $66.9 \%$ of cases of adult lumbar scoliosis went unreported on lumbar spine MR imaging studies. We expected our study to show similar results.

This study focuses on determining the prevalence of cervical ribs by using $\mathrm{CT}$, as opposed to radiographs, which have been more commonly used in the prior literature. We hypothesized that our results would fall between the minimum $(0.05 \%)^{5}$ and maximum $(3.0 \%)^{6}$ of the previously reported prevalences on the basis of radiographic findings. On the basis of previous experience with lumbar spine scoliosis ${ }^{9}$ at $66.9 \%$, we hypothesized that, when cervical ribs were present, there would be a $25 \%$ rate of underreporting by neuroradiologists.

\section{Materials and Methods}

Five thousand consecutive cervical spine CT scans obtained between November 2009 and May 2011 were compiled for viewing. The scans originated from all patients, regardless of their presenting symptoms, who had been to the inpatient and outpatient imaging services of a major university practice. Thin $0.5-\mathrm{mm}$ section CT scans were acquired in all cases. Coronal and sagittal 3-mm reconstructions were created by the CT technicians, but because all raw data were presented, oblique off-axis images were reconstructed as needed. The scan parameters used were section thickness of $0.5-0.75 \mathrm{~mm}$ with coronal and sagittal reconstructions, by using $120 \mathrm{kV}$ (peak) and 300 $\mathrm{mAs}$, on a Sensation 64-detector row scanner (Siemens, Erlangen, Germany).

If patients had repeat scans, were younger than 18 years of age, or 


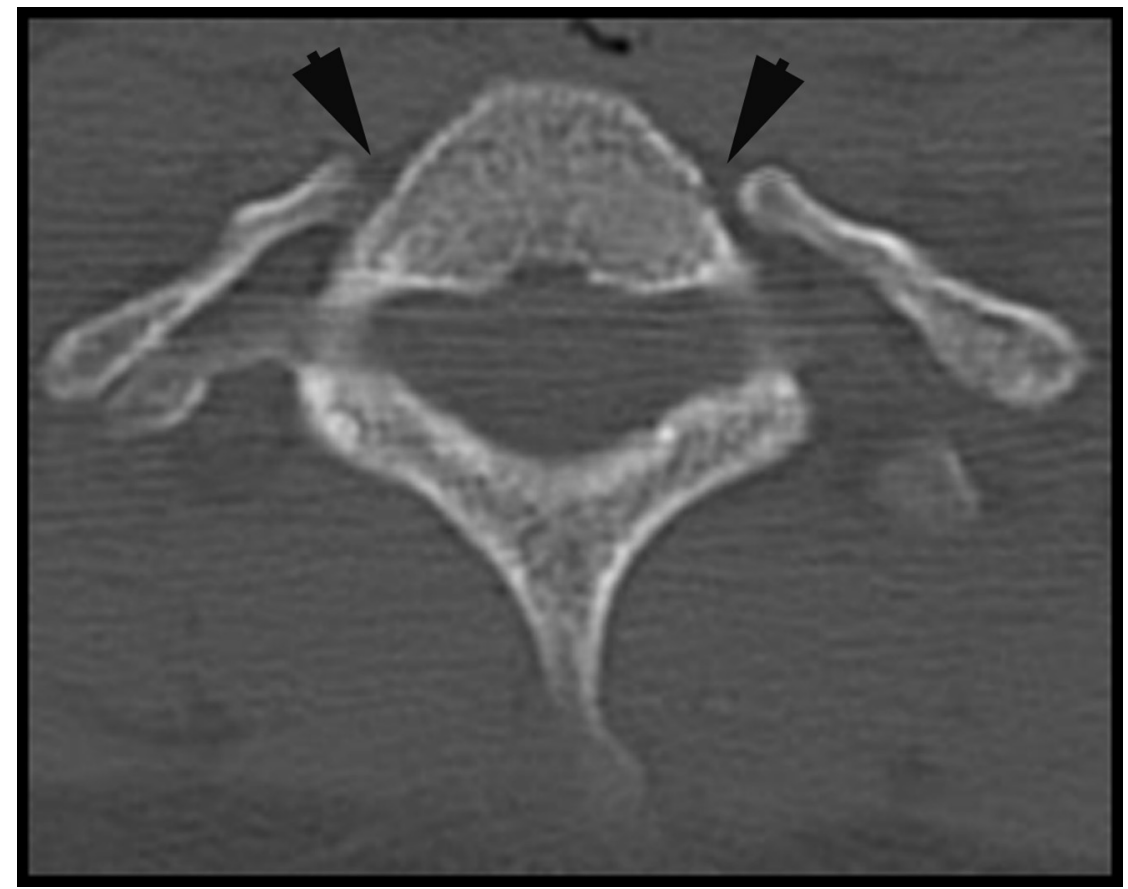

Fig 1. Axial CT image of bilateral cervical ribs.

had nondiagnostic-quality images, their corresponding examinations (1596) were eliminated from the study, yielding 3404 examinations for review. Five readers were trained for the project and were instructed to look exclusively for cervical ribs. The criteria for cervical ribs were defined as in several previous studies. ${ }^{2,4,10}$ These were as follows:

1) The cervical rib must articulate with the $C 7$ vertebra with a well-defined joint; if the rib was fused with the vertebra, it was considered an elongated transverse process.

2) The rib must not originate from the transverse process of the first thoracic vertebra, but rather the seventh cervical vertebral transverse process, which projects horizontally from the spine.

Any scan that the readers deemed to have a cervical rib was checked for accuracy by an expert reviewer with 23 years of subspecialty neuroradiology service. Figure 1 shows an example of bilateral processes that meet these criteria for cervical ribs. A separate data sheet was compiled containing all cross-sectional neuroradiology reports in the past decade, from May 31, 2001, to May 31, 2011, containing the words "cervical ribs."

In accordance with the Health Insurance Portability and Accountability Act, The Johns Hopkins University institutional review board reviewed and approved the protocol for this retrospective study and waived the requirement for informed consent.

\section{Results}

Of the 3404 studies included in this study, cervical ribs were found in 2.0\% (67/3404). The study included 1414 (41.5\%) women and $1990(58.5 \%)$ men. Women were found to have cervical ribs more than twice as often as men, $2.8 \%(39 / 1414)$ versus $1.4 \%(28 / 1990)$.

Ninety-four cervical ribs in 67 patients were found. Moreover, $59.7 \%(40 / 67)$ of patients with cervical ribs had a unilateral rib, while the remaining $40.3 \%$ (27/67) of patients had bilateral cervical ribs.
Underreporting of the presence of cervical ribs was a major focus of this study due to the speculation that it might be an issue. In fact, $74.5 \%$ (70/94) of the cervical ribs found in patients included in this study were not mentioned in the report made by the radiologist. This translates to underreporting in $50(74.6 \%)$ of the 67 patients. Based on the medical records of these reported studies, the prevalence of cervical ribs in the population included here would have been underestimated at $0.5 \%(17 / 3404)$ instead of $2.0 \%$.

The patients included in this study underwent CT scans for a variety of reported reasons, the most common being a form of trauma. Thoracic outlet syndrome was listed as the reason for obtaining the CT scan in $0.7 \%$ (24/3404) of patients; of those patients, $8.3 \%(2 / 24)$ were found to have cervical ribs.

Of the 49,929 radiology reports corresponding to MR imaging and CT examinations of the cervical spine created between 2001 and 2011 at our institution, the term "cervical rib" was found in just 271 studies $(0.5 \%)$. This prevalence of reported cervical ribs matches the erroneous radiology-report prevalence observed in our selected group of consecutive CT examinations performed between November 2009 to March 2011 (see above).

The prevalence of cervical ribs varied between races. In the 2 races comprising most our study population, African Americans and whites, cervical ribs were more than twice as prevalent $(2.8 \%, 47 / 1706)$ in the former than in the latter $(1.3 \%$, $18 / 1402)$.

\section{Discussion}

Previous studies have reported a wide range of values for the prevalence of cervical ribs in various populations, from $0.05 \%$ to $3.0 \% .^{5,6}$ Recently, Brewin et $\mathrm{al}^{2}$ examined 1352 chest radio- 
graphs and found a $0.74 \%$ rate of prevalence in a mixed sex and ethnicity population in London.

Individuals with a cervical rib are 10 times more likely to develop TOS. ${ }^{11}$ Although TOS is a frequently overlooked syndrome due to the difficulties associated with its diagnosis, those affected can experience pain, numbness, and/or tingling as a result of the compression of the brachial plexus or subclavian artery. ${ }^{7,12}$ The diagnosis of thoracic outlet syndrome is largely a clinical one. Putting the patients through provocative maneuvers clinically may be of more value that doing so during imaging. However such provocative maneuvers (scanning with the arms up and rotated outward) may lead to the demonstration of temporary occlusion/compression of the subclavian artery and/or compression of the brachial plexus. If the arterial pulses show diminution with such maneuvers, thoracic outlet syndrome is suspected. Management options for TOS caused by cervical ribs have included rib resections and anterior scalenectomies. ${ }^{13}$ Cervical ribs have also been associated with congenital brachial plexus palsy and brachial plexopathy. ${ }^{14,15}$

We determined the prevalence of cervical ribs on CT examinations at a university hospital located in a major city to be $2.0 \%$. As in prior studies, we determined the prevalence to be greater in women than in men. ${ }^{2,5}$ Race also appears to be a factor in determining the prevalence in a large population, with African Americans having twice the rate of whites.

As expected, our results indicate that cervical ribs are underreported by neuroradiologists. Without a clinical indication of possible thoracic outlet syndrome, neuroradiologists may not consider the supernumerary rib a critical finding related to the patient's care. Cervical spine CT scans are frequently ordered through the emergency department in the setting of trauma. This normal variant may not be readily considered in fracture detection, yet it may certainly be a source of neck and arm pain. Therefore, enhanced attention as to whether there is an articulating bony structure attached to C7 (ie, a rib) as opposed to an elongated transverse process on the thin-section source images and considering a cervical rib as a source of upper extremity paresthesias, pain, and motor complaints will assist in making this diagnosis. When needed, coronal reconstructions can help visualize the cervical rib-vertebral body articulation. Our study found that cervical ribs go unreported $74.6 \%$ of the time. In a patient with neck and upper extremity discomfort or sensorimotor symptoms, thoracic outlet syndrome and/or brachial plexopathy are important differential considerations. Therefore, the level of scrutiny for this anatomic variant must be heightened to prevent underdiagnosis of cervical ribs as a causative factor.

The implications of this study are that performance improvement in the detection of cervical ribs is warranted and may benefit from a practice quality-improvement initiative. Our conclusions point to a $2.0 \%$ prevalence of cervical ribs in a large heterogeneous population. This rate was obtained by using cross-sectional bone-targeted imaging, which, when used properly, is likely to be far more accurate than radiographs.

A limitation associated with this study stems from the ambiguity in distinguishing a cervical rib from an elongated transverse process. Although positive findings on scans were double-checked by an expert reviewer for accuracy, it is possible that some cervical ribs were mistaken for elongated transverse processes (and vice versa) by the initial reviewer. In addition, some CT scans were difficult to read due to movement by the patient during the examination with streak artifacts and noise arising from patients' shoulders at the C7-T1 level. Although readers were instructed to eliminate unreadable scans from the study, it is possible that scans including insufficiently visualized cervical ribs were included. These 2 limitations would result in our underestimating the true prevalence of this variant. Additionally, the racial makeup of the major city where our university hospital is located may affect the relevance of our results to populations that are racially dissimilar. This was a retrospective study in patients who had symptoms referable to their neck and spine, leading to ascertainment bias that limits the generalization of our results to the population at large. Yet, this limitation is not particularly meaningful because our conclusion that the abnormality is underreported was only applicable to the population that underwent CT scans; thus, this population could only include patients with symptoms referable to the neck and spine in any case.

\section{Conclusions}

In keeping with our hypothesis, cervical ribs are a relatively common condition in routine radiologic practice $(2.0 \%$ of adults affected) and are greatly underreported on cervical spine CT scans $(74.6 \%$ missed). It is important to recognize and report these common osseous anomalies, given their association as one of the causative factors of TOS and brachial plexopathy. Therefore, we recommend greater scrutiny in the radiologic evaluation of cervical spine CT scans with particular attention paid to the identification of cervical ribs, which may be the cause of a patient's symptoms.

Disclosures: Allan Belzberg-UNRELATED: Expert Testimony. law firms, Comments: I provide expert review in medical malpractice litigation, almost always defense, related to spine and peripheral nerve cases. David M. Yousem-UNRELATED: Royalties: Elsevier, Payment for Development of Educational Presentations: American College of Radiology.* *Money paid to the institution.

\section{References}

1. Bots J, Wijnaendts LC, Delen S, et al. Analysis of cervical ribs in a series of human fetuses. J Anat 2011;219:403-09

2. Brewin J, Hill M, Ellis $\mathrm{H}$. The prevalence of cervical ribs in a London population. Clin Anat 2009;22:331-36

3. Galis F. Why do almost all mammals have seven cervical vertebrae? Developmental constraints, Hox genes, and cancer. J Exp Zool 1999;285:19-26

4. Merks JH, Smets AM, Van Rijn RR, et al. Prevalence of rib anomalies in normal Caucasian children and childhood cancer patients. Eur J Med Genet 2005;48:113-29

5. Gulekon IN BC, Turgut HB. The prevalence of cervical rib in Anatolian population. Gazi Med J 1999;10:149-52

6. Steiner HA. Roentgenologic manifestations and clinical symptoms of rib abnormalities. Radiology 1943;40:175-78

7. Roos DB. Congenital anomalies associated with thoracic outlet syndrome: anatomy, symptoms, diagnosis, and treatment. Am J Surg 1976;132:771-78

8. Atasoy E. Thoracic outlet compression syndrome. Orthop Clin North Am 1996;27:265-303

9. Anwar Z, Zan E, Gujar SK, et al. Adult lumbar scoliosis: underreported on lumbar MR scans. AJNR Am J Neuroradiol 2010;31:832-37

10. Etter L. Osseous abnormalities of the thoracic cage seen in forty thousand 
consecutive chest photoroentgenograms. AJR Am J Roentgenol 1944;51: $359-63$

11. Sanders RJ. Thoracic Outlet Syndrome: Common Sequela of Neck Injuries. Philadelphia: J.B. Lippincott \& Co; 1991

12. Povlsen B, Belzberg A, Hansson T, et al. Treatment for thoracic outlet syndrome. Cochrane Database Syst Rev 2010;CD007218
13. Brooke BS, Freischlag JA. Contemporary management of thoracic outlet syndrome. Curr Opin Cardiol 2010;25:535-40

14. Becker $\mathrm{MH}$, Lassner $\mathrm{F}, \mathrm{Bahm} \mathrm{J}$, et al. The cervical rib: a predisposing factor for obstetric brachial plexus lesions. J Bone Joint Surg Br 2002;84:740-43

15. Desurkar A, Mills K, Pitt M, et al. Congenital lower brachial plexus palsy due to cervical ribs. Dev Med Child Neurol 2011;53:188-90 